\title{
Natural products chemistry and phytomedicine research in the new millennium: new developments and challenges
}

\author{
H. Wagner \\ Department of Pharmacy, Center of Pharma-Research, Institute of Pharmaceutical Biology, \\ University of Munich, Butenandstrasse 5, D-81377 Munich, Germany \\ E-mail: H.Wagner@cup.uni-muenchen.de
}

Dedicated to Professor S. Antus on the occasion of his $60^{\text {th }}$ birthday

(received 17 Dec 03; accepted 03 May 04; published on the web 06 May 04)

\begin{abstract}
The rationalisation of the new multidrug and multitarget concept of therapy in classical medicine will have also great implication on the future basic research in phytomedicine and an evidence based phytotherapy. It requires a concerted cooperation between phytochemists, molecular biologists, pharmacologists and clinicians with the aim to use modern high-tech methods for the standardisation of phytopreparation, to integrate new molecular biological assays into the screening of plant extracts and plant constituents and to increase the efforts into the efficacy proof of phytopreparations by controlled clinical trials which should be paralleled or followed pharmacocinetic and bioavailability studies. One major concern will be to investigate the multivalent and multitarget actions of plant constituents and standardized extracts with the aim of rationalizing the therapeutic superiority of many plant extracts over single isolated constituents. The new strategies are demonstrated on several examples of the own research projects and those of other laboratories.
\end{abstract}

Keywords: Evidence based rational phytotherapy, molecular biological approach, multidrug and multitarget phytotherapy

In the last ten years two revolutionary shifts occurred in classical medicine, which will have great implication also on the research in Phytomedicine and Natural Products Chemistry.

- The first change can be described as a gradual withdrawal from the dogma of monosubstance therapy and an increasing transition to the treatment of patients with drug combinations, as performed at present for the treatment of acquired immune deficiency syndrome (AIDS), cancer, malaria or hypertension. We call this therapy multidrug therapy.

- The second paradigm shift can be characterised as transition to a new kind of multitarget therapy, which is directed primarily toward the activation of defence, protective and repair 
mechanisms of the body rather than toward the direct destruction of the damaging agents (e.g. the tumour cell or the pathogenic microorganism).

Neither of these therapeutic strategies proposed at present by the new generation of clinicians and pharmacologists is absolutely new. Phytotherapy has long followed and developed these strategies by using mono-extracts or extract combinations containing mixtures of bioactive compounds and by activating primarily self-healing and protective processes of the human body, rather than attacking and directly destroying the damaging agents. These strategies are based on therapeutic experiences and the consideration that a complex pathophysiological process can be influenced more effectively and with fewer or less side-effects by a combination of several lowdosage compounds or the corresponding extracts than by a single highlydosaged isolated compound.

The rationalisation of both new concepts and strategies, however, requires scientific evidence. This enormous task, however, can be mastered only in a concerted cooperation between phytochemists, molecular biologists, pharmacologists and clinicians.

The efforts should focus on three research areas:

- application of all available modern, high-tech methods to standardise phytopreparations before conducting systematic pharmacological and clinical investigations

- integration of new molecular biological assays into the screening of extracts and plant constituents to evaluate their exact pharmacological profiles, to elucidate the synergistic effects of the constituents of an extract, and thereby to gain a better understanding of the various mechanisms underlying these pharmacological effects

- controlled clinical studies paralleled or followed by pharmacokinetic and bioavailability studies.

Owing to the enormous progress of specific high-tech analytical methods, we are able today to analyse even complex composed extracts and phytopreparations and to quantify the major active compounds, which are supposed to be responsible for the efficacy of an extract. The successful three-dimensional - high-performance liquid chromatography (HPLC) fingerprint analysis of a multiple extract combination of Kampo medicine prepared from eight herbal extracts and the quantification of their major constituents, illustrate the effectiveness of these modern tools. ${ }^{1}$ At the same time, these methods meet the quality standards of drug authorities and the reproducibility of pharmacological studies, along with the performance of good clinical practice (GCP), conforms to clinical trials requirements.

The following examples will explain the molecular biological screening approach in more detail. Most of the models operate on a receptor, signal transduction or genetic basis.

\section{Example 1}

In an attempt to rationalise the cardiotonic activity of hawthorn (Crataegus oxyacantha) extract preparations, verified by numerous clinical studies, it was found that procyanidins and flavone 
C-glycosides appear to be the main constituents of the herb responsible for the therapeutically relevant cardiotonic activity. ${ }^{2}$ Recently, we have reported that a hawthorn extract containing procyanidins and flavonoids, exhibits in vitro an angiotensinconverting enzyme (ACE)-inhibiting effect, ${ }^{3}$ which, together with the endothelin-dependent, smooth-musclerelaxing effect found recently in an aorta model, ${ }^{4}$ might account for the dilating effect on coronary vessels and a simultaneous reduction in blood pressure. Because the flavonoids also act as antioxidants, and cyclooxygenase and 5-lipoxygenase-inhibitors and have a thrombocytes aggregation reducing effect, it is necessary to produce special hawthorn extracts which contain both active classes of compounds in enriched form and standardised in the content of these procyanidins and flavone C-glycosides. In conclusion, we can state that the pharmacological multivalence of hawthorn constituents justifies the therapeutic application of hawthorn for the treatment of heart insufficiency grades I and II.

\section{Example 2}

Because the pharmacological role of garlic (Allium sativum) in the prevention and treatment of atherosclerosis has received increasing attention, we decided to investigate ajoene and allicin, two major constituents of fermented garlic juice, in a suitable molecular biological assay. It could be shown that both compounds inhibit the expression of inducible nitric oxide (iNO) synthase in activated macrophages. Because it is known that the inflammatory environment in human atherosclerotic lesions results in an expression of the inducible form of nitric oxide synthase $^{5}$ and, subsequently, in the formation of peroxynitrite, thereby aggravating the atherogenic process, these results may provide an interesting basic contribution with regard to the beneficial effects claimed for garlic in atherosclerosis prophylaxis. If we consider all the other known pharmacological effects caused by garlic preparations, including thrombocyte aggregation-inhibiting, anti-inflammatory, antioxidant triglyceride-decreasing and cholesteroldecreasing effects, garlic can be designated as one of the most notable examples of a multivalent herbal drug, in which the major bioactive constituents reveal synergistic, cardiovascular directed effects.

\section{Example 3}

This example describes the attempt to evaluate and rationalise the pharmacological profile and therapeutic potential of a multi-extract preparation from traditional Chinese medicine (TCM). The research project, headed by Prof. Sucher of the University Hong Kong, is the chemical and pharmacological investigation of a fixed herbal drug combination which has a great reputation in TCM for the prevention and therapy of stroke. ${ }^{6}$ The combination investigated consists of eight herbal drugs: root of Salvia miltiorhiza, rhizome of Ligusticum sinense, root of Paeonia rubra, root of Angelica pubescens, root of Stephania tetrandra, Ramulus Uncis of Uncaria rynchophylla, rhizome of Gastrodia elata and root of Panax ginseng. As a result of the detailed chemical and molecular-biological investigations the researchers were able to assign defined pharmacological effects and mechanisms of action to the individual herbal drugs and their major 
bioactive constituents. The individual TCM drugs were classified into four categories channelcollateral- stroke affecting, anti-inflammatory, antithrombotic and neuroprotective - and described as improving blood circulation, increasing cerebral blood flow and protecting the brain from ischaemic and reperfusion injuries. ${ }^{6}$

Meanwhile, many other herbal drug extracts have been screened using new molecular biological in vitro assays. These include extract preparations of Hypericum, Ginkgo, Silybum, Harpagophytum or Vitex agnus-castus.

From all these results we can conclude:

- all herbal drug extracts investigated with these new methods were found to have much greater pharmacological potential and broader profiles than suggested previously.

- most of the additional pharmacological effects were found to be synergistically additive to the earlier evaluated pharmacological activities (this provides a more comprehensive and a better understanding of the mechanism of action of the plant extracts).

- all investigations have shown that most of the extracts and individual constituents thereof exert multivalent or pleiotropic pharmacological effects (this multivalence of pharmacological activities can generate additive or overadditive, potentiated synergistic effects).

It is a rule in classical pharmacology that if two bioactive substances of a mixture have the same pharmacological target, an additive effect can be expected. If, however, two or more substances of a mixture have different pharmacological targets, a pharmacologically synergistic effect may result that can be greater than expected for the individual substances taken together (provided that none of the substances in the mixture exerts an antagonising effect). ${ }^{7}$ Those doseresponse investigations with two-component containing mixtures of bioactive pure compounds can be carried out using the isobol method as proposed by Berenbaum. ${ }^{8}$ We have carried out such an in vitro experiment using the thrombocyte aggregation assay with various mixtures of ginkgolide A and B, two major constituents of Ginkgo biloba. From the concave-up isobol curve we have obtained, a typical potentiated synergistic effect could be deduced. ${ }^{9}$ It is plausible that the isobol method cannot be applied to herbal extract mixtures. Here, detailed in vitro or in vivo comparative investigations with single constituents or mixtures and extract fractions or whole extracts must be performed. Evidence supporting the occurrence of synergy within phytomedicines is accumulating and has been reviewed recently by Williamson. ${ }^{10}$

The synergistic effects that have been measured exceed the effects of single compounds, or mixtures of them at equivalent concentrations, by a factor of two to four, or more. These synergistic additive or potentiated effects are of interest only if they can be verified by clinical studies. The next two examples may be instructive. 


\section{Example 4}

The Cannabis sativa herb has received much attention recently because of the detection of an endogen cannabinoid system in some parts of the human brain and the immune system, represented by the two cannabinoid receptors CB1 and CB2. New molecular biological investigations resulted in the identification of arachidonylethanol- amide (anandamide) and 2arachidonyl-glyzerol (ether) as endogen CB-ligands. According to current knowledge, this endogenic cannabinoid system plays an important role in the development of memory, in pain transduction and inhibition, in control of appetite, in lactation, in generation of emesis and as an immunomodulator. Among the most conspicuous pharmacological effects of $(-)$ trans- $\Delta^{9}-$ tetrahydrocannabinol (THC), which came onto the drug market under the drug name Dronabinol, $^{11}$ the muscle-relaxant, appetite stimulating and analgesic effects are the most interesting.

Meanwhile the idea of using THC as pure compound for the treatment of MS-patients has been given up after a comparative study with Cannabis has shown that the extract exhibited a much better antispastic activity than the THC substance alone, as measured in an immunogenic model of multiple sclerosis (MS). ${ }^{12}$ At present over 1\% of MS patients take Cannabis illegally for amelioration of spasticity and the pain associated with this condition. ${ }^{13}$ The reason for this better effect is probably due to the content of cannabidiol (CBD), in the extract, which amplifies the antispastic effect markedly and simultaneously reduces the undesirable psychotropic side effects of THC. It is not yet clear how this amplifying antispastic effect can be explained on a molecular basis, but there are some indications that CBD increases the permeation of THC into the muscle cells.

\section{Example 5}

The best clinical evidence for existing therapeutic synergism can be demonstrated by results obtained in a clinical trial performed with a standardised Hypericum extract in comparison with the synthetic psychopharmacon Imipramine in the treatment of patients with moderately severe depression. ${ }^{14}$ The Hypericum extract was administered over 6 weeks in a dose of 500mg extract per day, which corresponds to about 8-10mg bioactive compounds together (hypericins, hyperforin, xanthons, flavonoids, procyanidine). Imipramine was given in a concentration of 150 $\mathrm{mg} /$ day. The efficacy was measured by the procentual reduction of the HAMD-score values after 6 weeks' treatment. The results of the comparative study show that 8-10mg bioactive constituents of the Hypericum extract together must be therapeutically equivalent to $150 \mathrm{mg}$ imipramine. Interestingly, the Hypericum treatment has far fewer side-effects than the synthetic drug. ${ }^{7}$ The reason for this therapeutic equivalence of the medications has to be interpreted as a potentiated synergistic effect caused by the combination of Hypericum constituents, which are suggested to have different molecular targets in the brain. The possibility that the increased efficacy could be explained only due to an enhanced resorption rate of the constituents and thereby improved bioavailability, because of additional non-bioactive byproducts of the Hypericum extract, is not very likely and can be ruled out. 
At present, more than 400 controlled, mono- and double-blind, placebo-controlled clinical trials have been performed, which meet all international requirements of performance and efficacy. About $80 \%$ of the existing studies have been made with standardised versions of longused mono-extracts, including Ginkgo, Hypericum, Harpagophytum, Valeriana, Sabal, Urtica, Aesculus hippocastanum., Crataegus, Silybum or Echinacea. About 20\% were tested against chemosynthetic drugs, showing therapeutic equivalence of both medications at the same indication. Clinical evidence is available for the therapeutic superiority of Salix root extract over an isolated single constituent thereof ${ }^{15}$ and for the herbal drug combinations Valeriana/Piper methysticum $^{16}$ and Ginkgo/ginseng ${ }^{17}$ over only one of the two herbal drug combinations. It has to be emphasised that the concentrations of all bioactive constituents of a standardised extract applied together in these comparative studies, per day, were much lower than the applied amounts of the synthetic drug. This special dosage-effect relationship accounts for synergistic effects and at the same time for the absent or minimised side-effects described for these phytopreparations. The new strategy of multitarget phytotherapy is explained in the following two examples of cancer and hepatitis B/C therapy.

\section{Example 6}

The standard drug therapy for cancer in classical medicine uses cytostatic chemotherapeutics, which at ideal conditions arrest or destroy tumour cells. Most of the cytostatic drugs possess severe side-effects and reduce the quality of life. Apart from some exceptions, these drugs are not able to heal cancer patients or to extend their life span by more than 5 or 10 years. The new strategy follows a quite different concept. It aims at the stimulation of defence and repair mechanisms of the healthy tissues (e.g., induction of apoptosis, inhibition of angiogenesis, stimulation of heat-shock proteins and oncogen-suppressor genes). This multitarget therapeutic concept requires a cocktail of several individual or multivalent drugs, which, in a concerted and synergistic way, might be able to arrest the tumour growth.

Many laboratories worldwide work on the development of such a multidrug and multitarget concept.

\section{Example 7}

The standard therapy to treat hepatitis $B+C$ uses interferon- $\beta$ and/or some chemotherapeutic drugs such as ribaverine or lamivudine. The disadvantages are that the response rate for interferon is only $50-60 \%$ and the resistance rate for AZT and analogues is very high. The healing chances are limited. More success is expected with the new strategy of stimulating the immune system and repair mechanisms concertedly, to protect the healthy liver cell with antioxidants and inhibit apoptosis, as well as inflammatory and fibrotic processes. The strategies explained in these two examples and the development of adequate and effective drug combinations without severe side-effects are still in their infancy. The key for the development of new drugs is the use of molecular biology, with its new assays on a molecular level, to find ways to interfere with the different pathophysiological processes for an effective causative 
therapy. With these tools in hand, phytomedicine research has a good chance of contributing to the achievement of this goal.

\section{Summary}

Two new paradigm shifts in medicine characterise the beginning of the twenty-first century: the gradual renunciation of the long-standing reliance on monosubstance therapy in favour of a multidrug therapy, and the transition to a new kind of multitarget therapy, through which the interference of drugs with protective-, repair- and immunostimulatory mechanisms of the human body, rather than with single disease causing agents, gains more and more importance. Phytomedicine research has a good chance of contributing to these new strategies through the development of new and better drugs for an evidence-based and rational therapy. Great efforts in three research areas, however, are compulsory: (1) the increase in efforts to develop suitable standardisation methods for phytopreparations; (2) The integration of molecular biological assays into the screening of plant extracts, single isolated compounds thereof and phytopreparations; and (3) The performance of further placebo-controlled, mono- or doubleblind, clinical trials, paralleled or followed by pharmacokinetic and bioavailability studies. One major concern will be to investigate the multivalent and multitarget actions of plant constituents and standardised extracts, with the aim of rationalizing the therapeutic superiority of many plant extracts over single isolated constituents.

Phytomedicine and chemosynthetic pharmaceutical research find themselves in a race to develop new medicines, with fewer or no side-effects, for therapeutic and preventive application in illnesses for which causality-based treatments are non-existent or imperfect.

\section{References}

1. Amagaya, S.; Iizuka, A.; Makino B et al. General pharmacological properties of Sho-seiryuto (TJ-19) extracts. Phytomedicine 2001, 8, 338.

2. Tauchert M. Efficacy and safety of Crataegus extract WS1 1442 in patients with chronic stable heartinsufficiency at NYHA class III heart failure. Am. Heart. J. 2002, 143, 910.

3. Wagner, H.; Elbl, G.; Lotter, H.; Guinea, M. Evaluations of natural products as inhibitions of angiotensin-Iconverting enzyme (ACE). Pharm. Pharmacol. Lett. 1991, 1, 15.

4. Franck, U.; Günther, B.; Vierling, W.; Wagner, H. Investigation of Cecropia and Crataegus extracts for their angiotensin-converting enzyme inhibitory and vasorelaxant activities. Phytomedicine 1996, 3, 93.

5. Dirsch, V. M.; Kiemer, A. K.; Wagner, H.; Vollmar, A. M.; Effect of allicin and ajoene, two compounds of garlic on inducible nitric oxide synthase. Atherosclerosis 1998, 139, 333. 
6. Gong, X.; Sucher N. J. Stroke therapy in traditional Chinese medicine (TCM): prospects for drug discovery and development. Phytomedicine 2002, 9, 478.

7. Wagner, H. New targets in phytopharmacology of plants; Ernst, E., Ed.; Herbal Medicine: A Concise Overview for Professionals; Butterworth- Heinemann: Oxford, 2000.

8. Berenbaum, M. What is synergy? Pharmacol Rev 1989, 41, 93.

9. Wagner, H.; Steinke, B. Synergy effects of a mixture of Ginkgolide A+B in a thrombocyte aggregation inhibiting assay. Phytomedicine 2004 in press.

10. Williamson EM. Synergy and other interactions in phytomedicines. Phytomedicine 2001, 8, 401.

11. Bruhn, C. Dronabinol der Wirkstoff im Hanf. Dtsch Apoth Ztg 2002, 142, 3057.

12. Baker, D.; Pryce, G. Croxford JL et al. Cannabinoids control spasticity and tremor in an animal model of multiple sclerosis. Nature 2000, 404, 84.

13. Williamson, E.M.; Evans, F.J. Cannbinoids in clinical practice. Drugs 2000, 60, 1305.

14. Woelk, H.; Comparison of St. John's wort and imipramine for treating depression: randomised controlled trial. BMJ 2000, 321, 536.

15. Schmidt, B.; Ludke, R.; Selbmann, H.K. et al. Efficacy and tolerability of a standardized willow bark extract in patients with osteoarthritis randomised, placebocontrolled, double blind clinical trial. Phytother. Res. 2001, 15, 344.

16. Wheatley, D. Stress induced insomnia treated with kava and valerian. Hum. Psychopharmacol. 2001, 16, 353.

17. Scholey AB, Kennedy DO. Acute, dose-dependent cognitive effects of Ginkgo biloba, Panax ginseng and their combination in healthy young volunteers: differential interactions with cognitive demand. Hum Psychopharmacol 2002, 17, 35. 